Special issue of the International Conference on Computational and Experimental Science and Engineering (ICCESEN 2014)

\title{
Effect of Aspect Ratio on Natural Convection in a Cavity with Wavy Walls
}

\author{
M. ARICI*, M. KAN AND H. KarABAY \\ Kocaeli University, Engineering Faculty, Mechanical Engineering Department, 41380 Kocaeli, Turkey \\ In this study, flow characteristics and natural convection in a cavity with two wavy walls is studied numerically. \\ The wavy walls of the cavity are isothermal and at different temperatures while other straight walls are adiabatic \\ The fluid considered inside the cavity is air having Prandtl number of 0.71 . A parametric study is conducted for \\ various aspect ratios $(1 \leq A R \leq 5)$, Rayleigh numbers $\left(10^{3} \leq R a_{H} \leq 10^{6}\right)$ and inclination angles $\left(0^{\circ} \leq \theta \leq 180^{\circ}\right)$. \\ Streamlines, isotherms and local and mean Nusselt numbers are presented to show the effect of the investigated \\ parameters on the flow field and heat transfer.
}

DOI: 10.12693/APhysPolA.128.B-197

PACS: 44.25.+f, 47.15.Rq, 47.55.P-, 47.20.Bp

\section{Introduction}

Natural convection in enclosures has drawn a great deal of attention due to its importance in wide range of industrial applications such as cooling of electronic components, design of solar collectors and energy efficient buildings. Natural convection in a wavy enclosure is found in many engineering applications, such as doublewall thermal insulation, underground cable systems, and cooling of micro-electronic devices. The roughened surface is an important parameter to enhance heat transfer in the cavity. Comparing with flat plate, although the average heat transfer rate per unit wetted surface for a wavy surface is smaller, the total heat transfer rate for a wavy surface is, in general, higher [1,2]. A similar conclusion was drawn in [3] where a numerical comparative study on free convection in wavy and flat-plate solar collectors is conducted. It was observed that heat transfer rate increases in the case of wavy enclosure than that of flat enclosure. Ashjaee et al. [4] investigated experimentally and numerically free convection heat transfer from vertical surfaces and stated that numerical results agree well with the experimental data. Adjlout et al. [5] studied the effect of hot wavy wall for differentially heat square cavity for different Rayleigh numbers, inclination angles and amplitudes. It was reported that the mean Nusselt number decreases comparing with the square cavity and an increase in the undulation number on the hot wall reduces the heat transfer rate for an inclination angle greater than $75^{\circ}$. Sabeur-Bendehina et al. [6] studied free convection in an inclined rectangular cavity for various aspect ratios for $R a=10^{5}$. They reported that the Nusselt number decreases as aspect ratio increases. A similar conclusion was drawn in [7] also. Dalal and Das [8] investigated numerically free convection in an enclosure with a wavy wall for different number of undulation, inclination an-

*corresponding author; e-mail: muslumarici@gmail.com gles, amplitudes and the Rayleigh numbers. They showed that the inclination angle affects the flow and heat transfer rate. With increase in amplitude, the average Nusselt number on the wavy wall is considerably high at low $R a$ number. Mahmud et al. [9] investigated free convection in an enclosure bounded by two isothermal wavy walls and two adiabatic straight walls for various wave ratios, aspect ratios and the Grashof numbers. They reported that the aspect ratio is the most important parameter and heat transfer enhances at lower aspect ratios for a certain value of the Grashof number.

The objective of the present study is to investigate the effect of aspect ratio together with the Rayleigh number and inclination angle on the flow and heat transfer characteristics in a cavity bounded with two isothermal side wavy walls and two adiabatic straight walls.

\section{Problem description and numerical method}

The problem considered is a two-dimensional heat transfer in a cavity bounded with two wavy walls and two straight walls. The geometrical features of the enclosure are shown in Fig. 1. Height of the wavy walls is $H$ and length of the side walls is $L$. Forms of the wavy walls are described as

$$
\begin{aligned}
& x_{\text {right }}=1+0.025(-1+\cos (6 \pi y)), \\
& x_{\text {left }}=0.05+0.025(-1-\cos (6 \pi y)) .
\end{aligned}
$$

As shown in the figure, right and left wavy walls are kept at constant temperatures $\left(T_{H}\right.$ and $T_{C}$, respectively) while other straight walls are insulated. No slip velocity boundary conditions are imposed on all solid surfaces. Parameters of interest are the Rayleigh number, inclination angle and aspect ratio. Cavity is filled with air whose Prandtl number is kept to be 0.71 . Flow is considered to be steady, laminar and incompressible. Properties of air are assumed to be constant. Buoyancy force is treated by the Boussinesq approximation. Governing equations, namely continuity, $x$-momentum, $y$-momentum and energy equations are presented as 


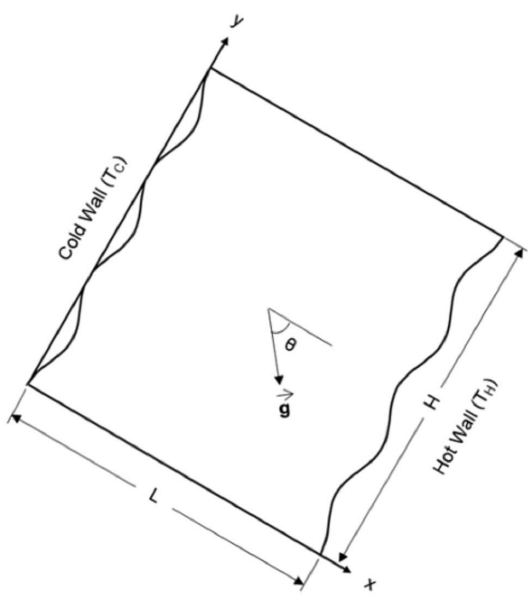

Fig. 1. Schematic representation of cavity with two wavy walls.

$$
\begin{aligned}
& \frac{\partial u}{\partial x}+\frac{\partial v}{\partial y}=0, \\
& u \frac{\partial u}{\partial x}+v \frac{\partial u}{\partial y}=-\frac{1}{\rho} \frac{\partial P}{\partial x}+v\left(\frac{\partial^{2} u}{\partial x^{2}}+\frac{\partial^{2} u}{\partial y^{2}}\right) \\
& \quad+g \beta\left(T-T_{\infty}\right) \sin \theta, \\
& u \frac{\partial v}{\partial x}+v \frac{\partial v}{\partial y}=-\frac{1}{\rho} \frac{\partial P}{\partial y}+v\left(\frac{\partial^{2} v}{\partial x^{2}}+\frac{\partial^{2} v}{\partial y^{2}}\right) \\
& \quad+g \beta\left(T-T_{\infty}\right) \cos \theta, \\
& u \frac{\partial T}{\partial x}+v \frac{\partial T}{\partial y}=\alpha\left(\frac{\partial^{2} T}{\partial x^{2}}+\frac{\partial^{2} T}{\partial y^{2}}\right) .
\end{aligned}
$$

A finite volume based software, ANSYS Fluent 15.0 is used to solve the governing equations. Convective terms in momentum and energy equations are discretized by second order upwind scheme. The SIMPLE algorithm is employed for velocity-pressure coupling. The convergence criteria for all governing equations are set to $10^{-6}$.

Nondimensional governing parameter Rayleigh number is defined as:

$$
R a_{H}=\frac{g \beta \Delta T H^{3}}{v^{2}} \operatorname{Pr}, \quad \operatorname{Pr}=\frac{\nu}{\alpha},
$$

where $g$ is the gravitational acceleration, $\beta$ is the thermal expansion coefficient, $\Delta T$ is the temperature difference, $H$ is the height of cavity, $\nu$ is the kinematic viscosity and $\alpha$ is the thermal diffusivity.

The mean Nusselt number is calculated at the hot wall of the cavity as

$$
N u=\frac{h H}{k} .
$$

Here, $k$ is the thermal conductivity of fluid and $h$ is the average heat transfer coefficient along the hot wall which is calculated from

$$
h=\frac{Q}{\left(T_{h}-T_{c}\right) L_{c}},
$$

where $Q$ is the total heat transfer from the hot surface.

A grid independence test is performed for different number of grid points. Grid pattern is refined until the variation of local Nusselt number became insignificant. It is concluded that 2100-10500 number of control volumes depending on aspect ratio and the Rayleigh number is suitable for this study. The numerical procedure is validated with the benchmark solution of De Vahl Davis [10] and Fusegi et al. [11]. As seen in Table, the numerical procedure shows a very good agreement with the benchmark solutions for entire range of the Rayleigh number where the discrepancy is less than $8 \%$.

\section{TABLE}

Comparison of $\mathrm{Nu}$ numbers obtained in the present study with those of benchmark solutions.

\begin{tabular}{l|c|c|c|c}
\hline \hline \multicolumn{1}{c|}{ Rayleigh number } & $10^{3}$ & $10^{4}$ & $10^{5}$ & $10^{6}$ \\
\hline De Vahl Davis [10] & 1.118 & 2.243 & 4.519 & 8.799 \\
Fusegi et al. [11] & 1.105 & 2.302 & 4.646 & 9.012 \\
present study & 1.1153 & 2.240 & 4.528 & 9.500
\end{tabular}

\section{Results}

The resulting flow and thermal structures are obtained for various aspect ratios $(1 \leq A R=H / L \leq 5)$, the Rayleigh numbers $\left(10^{3} \leq R a_{H} \leq 10^{6}\right)$ and inclination angles $\left(0^{\circ} \leq \theta \leq 180^{\circ}\right)$. Streamlines and temperature contours are presented in Fig. 2 for $A R=5$ at $R a_{H}=10^{5}$. As seen in Fig. 2a, pairs of large and small circulation cells occur side-by-side in the narrow wavy cavities. When the cavity is nearly horizontal the fluid in these circulation cells is hrld in the each pocket. Referring to the results in Fig. $2 \mathrm{~b}$ for $\theta \leq 30^{\circ}$, the air heated by the hot bottom surface rises up to the top cool surface and then falls down again in these circulation pockets. At the higher inclination angles, a main stream which circulates all around the cavity is observed. Thus, the heated air in the cavity cannot reach directly to the top cool surface and secondary cells appear in the central part of the cavity. For $\theta=180^{\circ}$, there are multiple circulation pockets occurring at the horizontal and vertical directions. However, the flow in these circulation cells is so weak that a thermal stratification occurs in the cavity.

Streamlines and isotherms for $A R=2$ at $R a_{H}=10^{5}$ are presented in Fig. 3. Comparing Fig. 2 and Fig. 3, it is seen that the flow structure at $A R=2$ are similar to that of $A R=5$ for the horizontal cases although the number of cells decreases. In the non-horizontal cases, the circulation cells are destroyed for $A R=2$ and a single air stream circulates in the whole cavity. Likewise to $A R=5$, for $\theta=180^{\circ}$ where top wall is hot and bottom wall is cold a thermal stratification behavior in the cavity is observed. A slightly distorted thermal strata appears for $\theta=150^{\circ}$ also since the buoyancy generated is not strong enough to overcome the resistance imposed by viscous forces.

The variation of local Nusselt numbers with inclination angle is plotted in Fig. 4. As seen in the figure, the Nusselt numbers for $A R=5$ are much higher than that of $A R=2$. Also, the maximum Nusselt values occurred for 

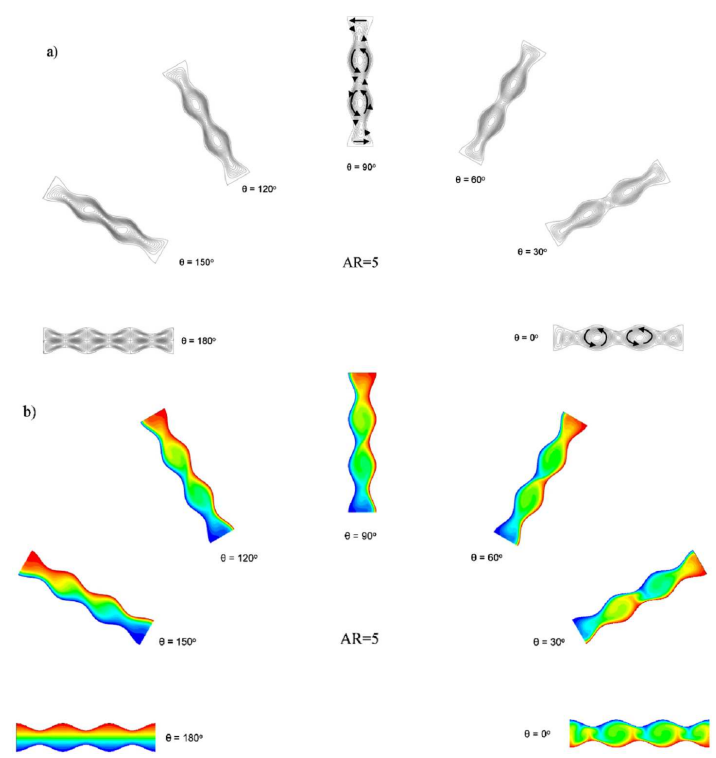

Fig. 2. a) Streamlines and b) temperature contours with different inclination angle for $A R=5\left(R a_{H}=\right.$ $\left.10^{5}\right)$.

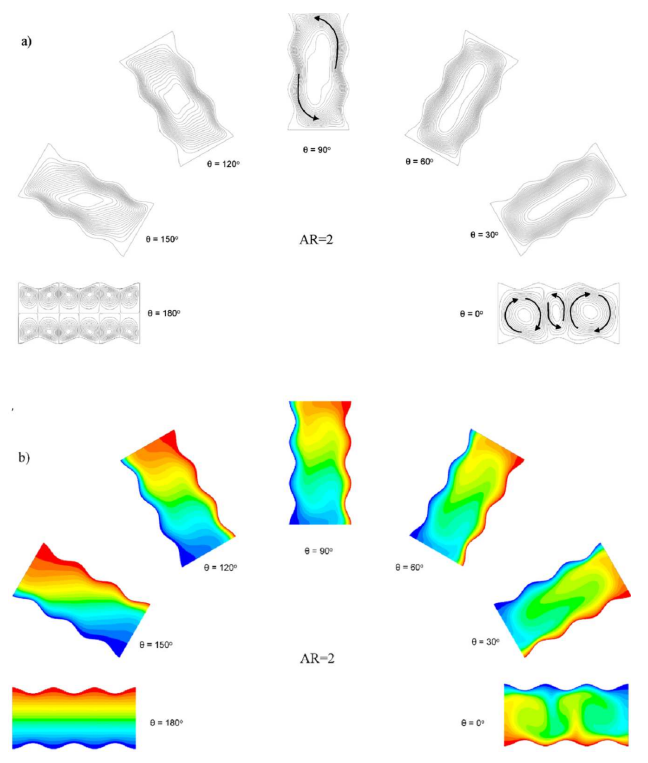

Fig. 3. a) Streamlines and b) temperature contours with different inclination angle for $A R=2\left(R a_{H}=10^{5}\right)$.

cases where the circulation pockets are observed. These circulation pockets create short-circuit air currents between the hot and cool surfaces which results in higher heat transfer rates. Variation of local Nusselt value shows peaks at these points. For the cases where circulation pocket does not appear, the hot air cannot reach directly to the cool surface and it is forced to circulate the whole cavity in a single stream which drops the heat transfer rates and lowers the Nusselt values.

The variation of mean Nusselt numbers with inclination angle is plotted in Fig. 5. As Rayleigh number in-

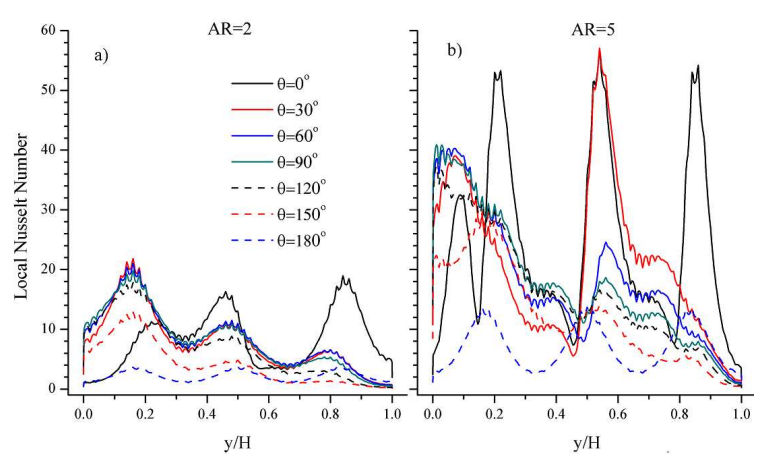

Fig. 4. Variation of local Nusselt number along the hot wavy wall at $R a_{H}=10^{5}$ a) $A R=2$, b) $A R=5$.

creases, the mean Nusselt number increases for all aspect ratios, as expected, except for $\theta=180^{\circ}$ where the heat transfer is dominated by conduction (see Figs. $2 \mathrm{~b}$ and $3 \mathrm{~b}$ ). It is interesting to note that although Rayleigh number increases from $10^{3}$ to $10^{4}$ for $A R=2$ and $\theta=0^{\circ}$, the mean Nusselt number does not change due to the fact that circulation cells do not onset yet. As seen in the figure, the inclination angle has almost no effect on the mean Nusselt number for $R a_{H}=10^{3}$ while it has a significant effect for higher $R a_{H}$ numbers. For $A R=1$ and $A R=2$, the inclination angle which maximizes the mean Nusselt number is $60^{\circ}$ at $R a_{H}=10^{4}$ and $10^{5}$ (except for $A R=1$ and $R a_{H}=10^{6}$ where it is $90^{\circ}$ ) while for $A R=5$ it is $30^{\circ}$ at $R a_{H}=10^{4}$ and $0^{\circ}$ at higher Rayleigh numbers.

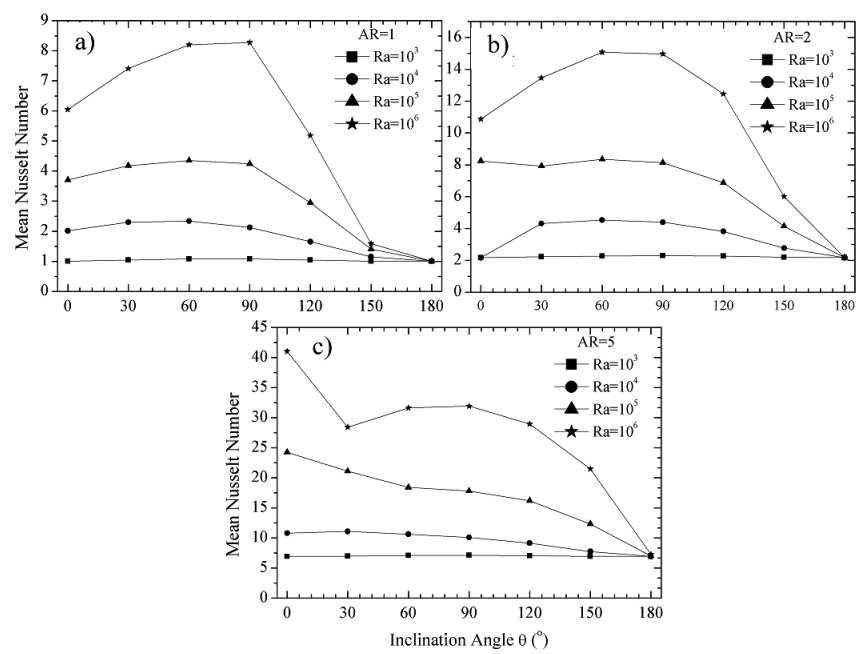

Fig. 5. Variation of mean Nusselt number with inclination angle a) $A R=1$, b) $A R=2$, c) $A R=5$.

\section{Conclusions}

In this study, flow characteristics and natural convection in a cavity bounded with two isothermal wavy walls and two insulated straight walls are studied numerically. 
A parametric study is conducted for various aspect ratios $(1 \leq A R \leq 5)$, the Rayleigh numbers $\left(10^{3} \leq R a_{H} \leq 10^{6}\right)$ and inclination angles $\left(0^{\circ} \leq \theta \leq 180^{\circ}\right)$. The computational results show that aspect ratio has a considerable effect on the flow structure and heat transfer. Increasing aspect ratio enhances the heat transfer. Inclination angle has also very significant effect on the flow field and heat transfer depending on the Rayleigh number and aspect ratio. The inclination angle where the heat transfer reaches a maximum decreases as aspect ratio increases.

\section{References}

[1] L.S. Yao, ASME J. Heat Transf. 105, 465 (1983).

[2] L.S. Yao, Int. J. Heat Mass Transf. 49, 281 (2006).

[3] Y. Varol, H.F. Oztop, Build. Environm. 43, 1535 (2008).
[4] M. Ashjaee, M. Amiri, J. Rostami, Heat Mass Transf. 44, 101 (2007).

[5] L. Adjlout, O. Imine, A. Azizi, M. Belkadi, Int. J. Heat Mass Transf. 45, 2141 (2002).

[6] A. Sabeur-Bendehina, O. Imine, L. Adjlout, Compt. Rend. Mecan. 339, 42 (2011).

[7] A. Sabeur-Bendehina, L. Adjlout, O. Imine, J. Appl. Sci. 3, 710 (2006).

[8] A. Dalal, M.K. Das, Int. J. Heat Mass Transf. 48, 3833 (2005)

[9] S. Mahmud, P.K. Das, N. Hyder, A.K.M.S. Islam, Int. J. Thermal Sci. 41, 440 (2002).

[10] G. De Vahl Davis, Int. J. Numer. Meth. Fluids 3, 249 (1983).

[11] T. Fusegi, J.M. Hyun, K. Kuwahara, B. Farouk, Int. J. Heat Mass Transf. 34, 1543 (1991). 